\title{
Inventario Taxonómico de Drosophilidae (Diptera) en el Parque Nacional Yasuni, Amazonia Ecuatoriana
}

\author{
Andrea Elizabeth ACURIO ${ }^{1}$, Violeta Lily RAFAEL ${ }^{2}$ \\ RESUMEN \\ En el Parque Nacional Yasuní, reconocido como un sector de alto endemismo y biodiversidad, ubicado al noroeste de la \\ Amazonía Ecuatoriana se realizó el inventario taxonómico de la familia Drosophilidae. Para la captura de los individuos se \\ utilizaron trampas con atrayente de banano y solución de levadura de cerveza. La identificación taxonómica se realizó usando \\ caracteres morfológicos y la terminalia de los machos. En total se colectaron 7425 individuos clasificados en 34 especies de \\ los géneros: Drosophila Fallén, 1823, Scaptodrosophila Duda, 1923, Neotanygastrella Duda, 1923 y Zaprionus Coquillett, 1901. \\ Seis de estas especies son nuevos registros para el Ecuador: D. araicas Pavan \& Nacrur, 1950, D. equinoxialis Dobzhansky, \\ 1946, D. kikkawai Burla, 1954, D. subsaltans Magalhães, 1956, D. neocordata Magalhães, 1956 y D. peninsularis Patterson \\ \& Wheeler, 1942. Estos datos incrementan el número de especies registrados para el país y para la región amazónica.
}

PALABRAS-CLAVES: Inventario taxonómico, Drosophila, Neotanygastrella, nuevos registros, Zaprionus.

\section{Taxonomic Survey of Drosophilidae (Diptera) in the Yasuni National Park, Ecuadorian Amazon}

\begin{abstract}
In the Yasuni National Park, a place recognized as a hot spot biodiversity, located in the Northwestern Ecuadorian Amazon was made a taxonomic survey of the Drosophilidae. Individuals were collected using traps with banana and yeast as bait. Taxonomic identifications were made by morphologic characters and male genitalia analysis. We collected 7425 individuals of 34 species, from the genera: Drosophila Fallén, 1823, Scaptodrosophila Duda, 1923, Neotanygastrella Duda, 1923 y Zaprionus Coquillett, 1901. Six of them are new records for Ecuador: D. araicas Pavan \& Nacrur, 1950, D. equinoxialis Dobzhansky, 1946, D. kikkawai Burla, 1954, D. subsaltans Magalhães, 1956, D. neocordata Magalhães, 1956 and D. peninsularis Patterson $\&$ Wheeler, 1942. This data increase the number of species records to Ecuador and the Amazon Region.
\end{abstract}

KEY WORDS: Taxonomic Survey, Drosophila, Neotanygastrella, new records, Zaprionus.

1 Escuela de Ciencias Biológicas de la Pontificia Universidad Católica del Ecuador. E-mail: andreaacurio@yahoo.com

2 Escuela de Ciencias Biológicas de la Pontificia Universidad Católica del Ecuador. E-mail: vrafael@puce.edu.ec 


\section{INTRODUCCIÓN}

La Amazonía Occidental es reconocida como la parte más biodiversa de la cuenca amazónica (Finer et al., 2008), la extraordinaria riqueza de esta región ha sido documentada en estudios taxonómicos de plantas (Steege et al., 2003; Valencia et al., 2004), insectos (Erwin et al., 2004), aves (Stotz et al., 1996) anfibios (Young et al., 2004), y mamíferos (Ceballos y Ehrlich, 2006). Esta región además tiene una gran riqueza cultural debido a la variedad de grupos étnicos que alberga, muchos de los cuales son los últimos grupos en aislamiento voluntario. En Ecuador, la región amazónica está conformada por las provincias de Orellana, Pastaza, Napo, Sucumbíos, Morona Santiago, Zamora Chinchipe y se extiende sobre un área de $130.000 \mathrm{~km}^{2}$.

Entre las provincias de Napo y Orellana, se encuentra Yasuní, el parque nacional más grande del Ecuador, el cual fue reconocido internacionalmente por su importancia recibiendo la designación de Patrimonio Natural de la Humanidad y Reserva del Hombre y la Biosfera por la UNESCO en 1989. Este parque protege una región de extraordinaria biodiversidad, herencia cultural y vida silvestre prístina. Es considerado uno de los sitios más diversos del planeta (Gentry, 1993; Kress et al., 1998; Heyer et al., 1999; Pitman et al., 2001). Solo en una hectárea de bosque han sido encontradas más de 100.000 especies de insectos (Erwin et al., 2004).

Dentro del Parque Nacional Yasuní también existen grandes reservas petroleras que actualmente están siendo prospectadas y explotadas, incrementando el porcentaje de áreas deforestadas en el interior del Parque y generando consecuencias biofísicas negativas como la erosión del suelo, la contaminación de ríos y la polución del aire (Fearnside, 1990). Simultáneamente, la deforestación causada por la explotación maderera está acrecentando la migración y el establecimiento de nuevas poblaciones humanas, que acarrean problemas como la introducción de especies exóticas y la disminución de especies nativas, muchas de las cuales son desconocidas (UICN, 2006). Dentro de este contexto, los inventarios taxonómicos realizados en la Amazonía tienen una relevancia especial porque nos permiten conocer y cuantificar la biodiversidad de la región, además aportan información importante para comprender aspectos de la ecología, biogeografía y distribución geográfica de las especies.

Existe además, una necesidad urgente de encontrar indicadores biológicos efectivos que permitan evaluar el efecto de las intervenciones antropogénicas sobre el ecosistema (MacGeoch, 1998).

Los estudios sobre diversidad de la familia Drosophilidae en la región Amazónica son pocos (Martins 1989; 2001) sin embargo, han mostrado la influencia de la deforestación del bosque amazónico en la diversidad de drosofilídeos. En Ecuador la diversidad y el endemismo del género Drosophila es alta, esto lo demuestran trabajos realizados mayoritariamente en las regiones andina y costera (Rafael y Vela, 2000; Acurio y Rafael, 2009). La región amazónica no había sido explorada hasta el presente trabajo, convirtiéndose este en el primer inventario taxonómico de drosofilídeos realizado en la región amazónica occidental.

\section{MATERIALES Y MÉTODOS}

El Parque Nacional Yasuní está ubicado al noroeste de la Amazonía Ecuatoriana ( $0^{\circ} 39^{\prime} 03^{\prime \prime} \mathrm{S}$; $\left.76^{\circ} 22^{\prime} 42^{\prime \prime} \mathrm{W}\right)$, tiene una extensión cercana a un millón de hectáreas (Figura 1). El área de estudio es un Bosque Húmedo de Tierras Bajas, con un dosel de 15-30 m de altura y árboles emergentes que pueden alcanzar hasta $50 \mathrm{~m}$. No presenta estacionalidad en cuanto a lluvia y temperatura, tiene una precipitación media mensual de $235 \mathrm{~mm}$ (Valencia et al., 2004). La temperatura ambiental en este bosque varía de $22{ }^{\circ} \mathrm{C}$ (mínima) a $32^{\circ} \mathrm{C}$ (máxima) y la humedad ambiental varía del $56 \%$ al $96 \%$.

Las colectas fueron realizadas en cuatro diferentes niveles de disturbio antrópico, tomando en cuenta el grado de alteración, tipo de vegetación del sitio y cercanía del lugar a carreteras o poblados. Los sitios muestreados fueron: Sin Disturbios (D.0), Disturbio Bajo (D.1, D.2 y D.3), Disturbio Medio (D.4) y Disturbio Alto (D.5). Se realizaron colectas diarias durante 15 días en los meses de diciembre del 2005, marzo y julio del 2006. En cada uno de los seis puntos de muestreo se utilizaron seis trampas con una distancia mínima de $30 \mathrm{~m}$, totalizando 36 unidades de muestra. Las trampas consistían en botellas plásticas de $25 \mathrm{~cm}$ de largo $\times 5 \mathrm{~cm}$ de diámetro (ver más detalles en Rafael et al., 2000), que contenían $110 \mathrm{ml}$ de solución de banano Musa X paradisiaca L., y levadura de cerveza Saccharomyces cerevisiae, en una proporción de 3 a 1. Cada trampa fue colocada a $15 \mathrm{~cm}$ del suelo. Después de cada colecta, el atrayente de la trampa fue sustituido por uno nuevo para evitar un sesgo a favor de especies que tienen preferencia por trampas más viejas (Medeiros y Klaczko, 1999).

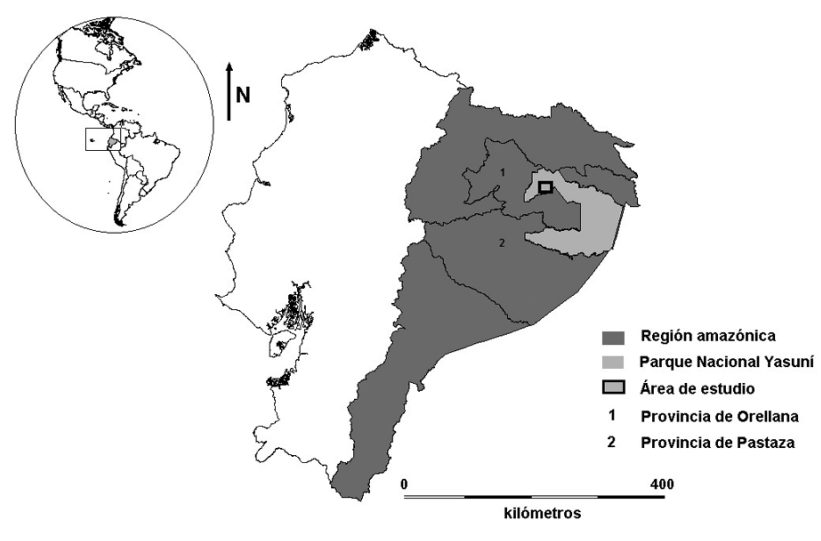

Figura 1- Mapa del Ecuador continental mostrando la localización del Parque Nacional Yasuni. 
Para evaluar preferencias en el tipo de sustrato, se colectaron frutos maduros que se encontraban en el piso del bosque, a una distancia no mayor a un metro de la trampa. Los frutos colectados se colocaron en frascos de vidrio de 15 $\mathrm{cm}$ de largo $\mathrm{x} 15 \mathrm{~cm}$ de diámetro con tapones de algodón, se trasladaron al Laboratorio de Genética Evolutiva en la ciudad de Quito (a $800 \mathrm{~km}$ de distancia del área de muestreo) y se mantuvieron a la temperatura promedio registrada en el sitio de colecta.

La identificación taxonómica se realizó mediante el análisis de los caracteres morfológicos y la terminalia de los machos. Las hembras colectadas, fueron colocadas individualmente en tubos de ensayo con medio de banano (isolíneas) para analizar la genitalia externa de un macho de su descendencia.

Para la identificación taxonómica de especies crípticas de los subgrupos melanogaster y willistoni además, se utilizaron caracteres morfológicos propuestos por Burla et al., (1949), Malogolowkin (1952), Shorrocks (1972) y Bächli et al. (2004).

Los especímenes de los individuos colectados en este estudio y sus terminalias contenidas en microtubos con glicerol puro fueron depositados en la Sección de Invertebrados del Museo de Zoología de la Pontificia Universidad Católica del Ecuador (QCAZ).

\section{RESULTADOS Y DISCUSIÓN}

Se identificaron 34 especies de drosofilídeos de un total de 7425 individuos colectados; estás especies pertenecen a cuatro géneros: Drosophila Fallén, 1823; Scaptodrosophila Duda, 1924; Neotanygastrella Duda, 1925 y Zaprionus Coquillett, 1901 (Tabla 1).

El 98\% de los individuos colectados pertenecen al género Drosophila representado por tres subgéneros: Drosophila Fallén, 1823; Dorsilopha Sturtevant, 1942 y Sophophora Sturtevant, 1939.

El subgénero más abundante fue Sophopora (95.7\%). El grupo de especies más abundante fue willistoni $(D$. willistoni Sturtevant, 1916; D. paulistorum Dobzhansky \& Pavan en Burla et al., 1949 y D.equinoxialis Dobzhansky, 1946). El grupo saltans registró el mayor número de especies, seis en total (D. emarginata Sturtevant, 1942; D. neocordata Magalhães, 1956; D. parasaltans Magalhães, 1956; D. prosaltans Duda, 1927; D. sturtevanti Duda, 1927; D. subsaltans Magalhães, 1956).

El porcentaje de larvas que llegaron al estadio adulto de los frutos colectados en el piso del bosque fue muy bajo (1.8\%). Este porcentaje se debe a la susceptibilidad de las larvas al ser transportadas lejos del sitio de colecta, muchas larvas murieron durante el transporte. Sólo Drosophila vicentinae

Tabla 1- Lista de las especies colectadas con trampas de banano y levadura durante diciembre 2005, marzo y julio del 2006 en seis sitios muestreados en el Parque Nacional Yasuní. Ecuador. (*) indica grupos de especie de Drosophila.

\begin{tabular}{|c|c|c|c|c|c|c|c|}
\hline \multirow{2}{*}{\multicolumn{2}{|c|}{ Familia Drosophilidae (34 especies) }} & \multicolumn{6}{|c|}{ Grado de disturbio } \\
\hline & & D.0 & D.1 & D.2 & D.3 & D.4 & D.5 \\
\hline \multicolumn{8}{|c|}{ Género Drosophila Fallén (31 especies) } \\
\hline \multicolumn{8}{|c|}{ subgénero Dorsilopha Sturtevant (1 especie ) } \\
\hline *busckii & D. busckii Coquillett & & & & 10 & & \\
\hline \multicolumn{8}{|c|}{ subgénero Drosophila Fallén (7 especies ) } \\
\hline * annulimana & D. araicas Pavan \& Nacrur & 2 & & & & 7 & \\
\hline \multirow[t]{2}{*}{${ }^{\star}$ cardini } & D. cardini Sturtevant & & & & & & 25 \\
\hline & D. cardinoides Dobzhansky \& Pavan & & 3 & & 7 & & 80 \\
\hline \multirow[t]{4}{*}{ *repleta } & D. mercatorum Patterson \& Wheeler & & & & 3 & & \\
\hline & D. paranaensis Barros & & 3 & & & & 10 \\
\hline & D. peninsularis Patterson \& Wheeler & 7 & 7 & & & 1 & \\
\hline & D. vicentinae Vilela & & 5 & & & & \\
\hline \multicolumn{8}{|c|}{ subgénero Sophophora Sturtevant (16 especies) } \\
\hline \multirow[t]{5}{*}{ *melanogaster } & D. ananassae Doleschall & & & & & 5 & 118 \\
\hline & D. kikkawai Burla & & & & & & 11 \\
\hline & D. malerkotliana Parshad \&Paika & & & & & & 161 \\
\hline & D. melanogaster Meigen & & & & & & 476 \\
\hline & D. simulans Sturtevant & & & & & & 248 \\
\hline \multirow[t]{3}{*}{ *saltans } & D. emarginata Sturtevant & 26 & & 2 & 6 & 2 & 3 \\
\hline & D. neocordata Magalhães & & & & & 5 & \\
\hline & D. parasaltans Magalhães & 13 & 19 & & 17 & & \\
\hline
\end{tabular}


Tabla 1- Continuación

\begin{tabular}{|c|c|c|c|c|c|c|c|}
\hline \multirow{2}{*}{\multicolumn{2}{|c|}{ Familia Drosophilidae (34 especies) }} & \multicolumn{6}{|c|}{ Grado de disturbio } \\
\hline & & \multirow{2}{*}{ D.0 } & \multirow[t]{2}{*}{ D.1 } & \multirow[t]{2}{*}{ D.2 } & \multirow[t]{2}{*}{ D.3 } & \multirow{2}{*}{$\begin{array}{c}\text { D. } 4 \\
4\end{array}$} & \multirow{2}{*}{$\begin{array}{c}\text { D.5 } \\
27\end{array}$} \\
\hline & D. prosaltans Duda & & & & & & \\
\hline & D. sturtevanti Duda & 117 & 152 & 122 & 210 & 132 & 89 \\
\hline & D. subsaltans Magalhães & & 2 & & 5 & 4 & \\
\hline \multirow[t]{5}{*}{ *willistoni } & D. equinoxialis Dobzhansky & 10 & 26 & 28 & 25 & 42 & 53 \\
\hline & D. nebulosa Sturtevant & & & & & & 30 \\
\hline & D. paulistorum Dobzhansky \& Pavan & 24 & 393 & 342 & 277 & 386 & 473 \\
\hline & D. sucinea Patterson \& Mainland & & & & & & 10 \\
\hline & D. willistoni Sturtevant & 56 & 474 & 471 & 473 & 1114 & 559 \\
\hline \multirow[t]{7}{*}{ sin agrupar } & Drosophila sp1 & & 1 & & & & \\
\hline & Drosophila sp2 & & 2 & 1 & & & \\
\hline & Drosophila sp3 & & & 1 & & & \\
\hline & Drosophila sp4 & & & & & & 4 \\
\hline & Drosophila sp5 & & & & & 1 & \\
\hline & Drosophila sp6 & & 1 & & & & \\
\hline & Drosophila sp7 & & & 2 & & & \\
\hline \multicolumn{8}{|c|}{ Género Neotanygastrella Duda (1 especie) } \\
\hline & Neotanygastrella. sp & & & & & & 1 \\
\hline \multicolumn{8}{|c|}{ Género Scaptodrosophila Duda (1 especie) } \\
\hline *latifascieformis & S. latifascieformis Duda & & & & & & 46 \\
\hline \multicolumn{8}{|c|}{ Género Zaprionus Coquillett (1 especie) } \\
\hline \multirow[t]{3}{*}{ *armatus } & Z. indianus Gupta & & & & & & 104 \\
\hline & & 255 & 1088 & 969 & 1033 & 1703 & 2377 \\
\hline & Total & & & & & & 7425 \\
\hline
\end{tabular}

Vilela, 1983 llegó al estadio adulto y emergió del fruto de Cabralea canjeriana (Vell.) Mart. (Meliaceae).

Las siete especies no identificadas encontradas en este estudio, fueron clasificadas dentro del género Drosophila, porque sus caracteres morfológicos corresponden a la definición del género encontrada en Bächli et al., (2004).

Los seis nuevos registros para Ecuador son:

\section{Drosophila araicas Pavan \& Nacrur, 1950}

Material examinado: 9 individuos. Provincia Francisco de Orellana. Parque Nacional Yasuní. 0041'15”W 76²2’22”S, $200 \mathrm{msnm}$, trampa de banano, A. Acurio. Julio 2006. (4웅) 7855QCAZ, 7913QCAZ, 7914QCAZ, 7915QCAZ; (4ふ઼う) 7856QCAZ, 7916QCAZ, 7917QCAZ, 7918QCAZ.

Nota: Pertenece al grupo annulimana Duda, 1927, esta especie ha sido reportada en Brasil (Val, 1982) y Venezuela (Hunter, 1970). Debido al parecido morfológico que tienen $D$. araicas y $D$. aracataca se compararon los genitalia masculinos, femeninos y los rasgos morfológicos de ambas especies.

Drosophila equinoxialis Dobzhansky, 1946
Material examinado: 158 individuos. Provincia Francisco de Orellana. Parque Nacional Yasuní. 0041'15”W 76²2'22"S, 200 msnm, trampa de banano, A. Acurio. Julio 2006. (1우으) 7911QCAZ; (1ठð) 7912QCAZ.

Nota: pertenece al grupo willistoni Burla, 1949. Es una especie común en la Región Neotropical, ha sido reportada en Brasil, Colombia, Guyana, Perú y Venezuela (Bächli, 2006). Es una de las cuatro especies gemelas del subgrupo willistoni distinguibles principalmente por los genitalia masculinos y femeninos (Burla et al., 1949, Malogolowkin, 1952) y análisis moleculares (García et al., 2006). Debido a la cercanía morfológica de $D$. equinoxialis con sus especies gemelas $D$. willistoni y $D$. paulistorum se compararon los genitalia externos masculinos, femeninos y rasgos morfológicos.

\section{Drosophila kikkawai Burla, 1954}

Material examinado: 11 individuos. Provincia Francisco de Orellana. Estación Científica Yasuní. 0041'28”W 76²4'04”S, $192 \mathrm{msnm}$, trampa de banano, A. Acurio. Marzo 2006. (3우) 7905QCAZ, 7906QCAZ, 7907QCAZ; (4ふ̋ふ) 7909QCAZ, 7910QCAZ, 7794QCAZ, 7908QCAZ. 
Nota: Pertenece al grupo melanogaster, originario de la Región Oriental, D. kikkawai es una especie cosmopolita (David y Tsacas, 1981) que fue reportada por primera vez en América del Sur en 1949 (Bock y Wheeler, 1972), posee dos peines sexuales característicos que son de gran ayuda en su identificación taxonómica.

\section{Drosophila subsaltans Magalhães, 1956}

Material examinado: 11 individuos. Provincia Francisco de Orellana. Parque Nacional Yasuní. 0041'15”W 76²2'22”S, $200 \mathrm{msnm}$, trampa de banano, A. Acurio. Julio 2006. (4우우 7894QCAZ, 7895QCAZ, 7896QCAZ, 7897QCAZ; (4ð̋ ठૈ) 7899QCAZ, 7900QCAZ, 7901QCAZ, 7902QCAZ

Nota: Pertenece al grupo saltans, de amplia distribución en la región Neotropical (Magalhães, 1962). D. subsaltans, ha sido reportado únicamente en Brasil (Bächli, 2006) y es morfológicamente cercana a $D$. parasaltans (Magalhães y Bjornberg, 1957) pero se diferencian por los genitalia masculinos.

\section{Drosophila neocordata Magalhães, 1956}

Material examinado: 5 individuos. Provincia Francisco de Orellana. Parque Nacional Yasuní. 0041'15"W 76²2'22”S, $200 \mathrm{msnm}$, trampa de banano, A. Acurio. julio 2006. $\left(1 \widehat{\partial}^{\widehat{\partial}}\right)$ 7929QCAZ.

Nota: pertenece al grupo saltans, ha sido registrada únicamente en Brasil (Bächli, 2006) es cercana morfológicamente a $D$. cordata pero se diferencian por los genitalia masculinos (Magalhães y Bjornberg, 1957).

Drosophila peninsularis Patterson \& Wheeler, 1942

Material examinado: 15 individuos. Provincia Francisco de Orellana. Parque Nacional Yasuní. 0041'15”W 76²2'22”S, $200 \mathrm{msnm}$, trampa de banano, A. Acurio. julio 2006. (4우우)

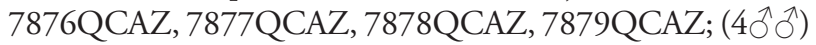
7859QCAZ, 7875QCAZ, 7880QCAZ, 7881QCAZ.

Nota: Pertenece al grupo repleta, endémico de la Región Neotropical. Esta especie ha sido reportada en los Estados Unidos e Indias Occidentales (Bächli, 2006). Este es el primer registro de esta especie para Sudamérica. $D$. peninsularis está relacionada a $D$. carcinophila, de la cual difiere por su número de dientes primarios del surestilo y el tamaño del edeago (Vilela, 1983).

Los datos de ocurrencia de especies obtenidos en este inventario taxonómico contribuyen a un mejor conocimiento de la diversidad de los drosophilideos en la Región Amazónica. Estos datos además son relevantes para la conservación de la Amazonía debido a que Drosophila es un bioindicador eficaz del estado del ecosistema (Acurio y Rafael en prensa). Estos dípteros tienen varias ventajas sobre otros insectos indicadores, por ejemplo: ciclos de vida cortos, sensibilidad extrema a cambios ambientales (Tidon, 2006), su movilidad que facilita la colonización de un hábitat (van der Linde, 1997; van der Linde y Sevenster, 2002) y son fáciles de colectar. La realización de inventarios taxonómicos de drosofilídeos corresponde a la primera etapa en la generación de conocimientos que servirá de base para el desarrollo de herramientas biológicas que permitan formular medidas de conservación eficientes para la región amazónica.

\section{AGRADECIMIENTOS}

Nuestros agradecimientos para: al Dr. Carlos Vilela y al Dr. Sergio Castrezana, por sus valiosos comentarios para la elaboración de este manuscrito, a la Dra. Laura Arcos Terán y a la Organización Idea Wild por la donación de materiales de colecta. Este trabajo fue financiado por la Pontificia Universidad Católica del Ecuador (PUCE) con el Proyecto D21-506021-C13034.

\section{BIBLIOGRAFÍA CITADA:}

Acurio, A.; Rafael, V. 2009. Diversity and geographical distribution of Drosophila (Diptera Drosophilidae) in Ecuador. Drosophila Information Service 92 (en prensa).

Bächli, G.; Vilela, C.R.; Andersson Escher, S.; Saura, A. 2004. The Drosophilidae (Diptera) of Fennoscandia \& Denmark. Fauna Entomologica Scandinavica. Vol. 39. Brill, Leiden. The Netherlands. 362 pp.

Bächli, G. 2006. Taxodros. Base de Datos de Taxonomía de Drosophilidae (http://www.taxodros.unizh.ch). Acceso 25/02/2006.

Bock, I.R.; Wheeler, M.R. 1972. The Drosophila melanogaster Species Group. The University of Texas Publication. Texas, USA 102pp.

Burla, H.; da Cunha, A.B.; Cordeiro, A.R.; Dobzhansky, T.; Malogolowkin, C.; Pavan, C. 1949. The willistoni group of sibling species of Drosophila. Evolution, 3: 300-314.

Ceballos, G.; Ehrlich, P.R. 2006. Global mammal distributions, biodiversity hotspots, and conservation. Proc. Natl. Acad. Sci. USA 103: 19374-19379

David, J.R.;Tsacas, L. 1981. Cosmopolitan, subcosmopolitan \& widespread species: different strategies within the Drosophilid family (Diptera). C. r. Seanc. Societe biogeog. 57: 11-26.

Erwin, T.L.; Pimienta, M.C.; Murillo, O.E.; Aschero, V. 2004. Mapping patterns of $\beta$-diversity for beetles across the western Amazon Basin: A preliminary case for improving conservation strategies. Proc. Calif. Acad. Sci. 56: 72-85.

Fearnside, P. M.1990. Environmental destruction in the Brazilian Amazon. En The future of Amazonia, editado por D. Goodman y A. Hall, 171-220. London: St. Martin's Press.

Finer, M.; Jenkins, C. N.; Pimm, S.L.; Keane, B.; Ross, C. 2008. Oil and Gas Projects in the Western Amazon: Threats to Wilderness, Biodiversity, and Indigenous Peoples. PLoS ONE 3(8): e2932. doi:10.1371/journal.pone.0002932 
García, A.C.L.; Rohde, C.; Audino, G.F.; Valente, V.L.S.; Valiati, V.H. 2006. Identification of the sibling species of the Drosophila willistoni subgroup through the electrophoretical mobility of acid phosphatase-1. J. Zool. Syst. Evol. Res., 44: 212-216.

Gentry, A. 1993. A Field Guide to the Families and Genera of Woody Plants of Northwest South America (Colombia, Ecuador, Peru) with Supplementary Notes on Herbaceous Taxa. Conservation International, Washington, DC, USA. 895 pp.

Heyer, W.R.; Coddington, J.A.; Kress, W.J.; Acevedo-Rodriguez, P.; Cole, D.; Erwin, T.L.; Meggers, B.J.; Pogue, M.G.; Thorington, R.W.; Jr.; Vari, R.P.; Weitzman, M.J. and Weitzman, S.H. 1999. Amazonian biotic data and conservation decisions, Ciência e Cultura, Sao Paulo, 51(5-6): 372-385.

Hunter, A.S. 1970. Drosophila of Venezuela. Drosophila Information Service, 45: 124.

Kress, J.; Heyer, Wr.; Acevedo, P.; Coddington, J.A.; Cole, D.; Erwin, T.L.; Meggers, B.J.; Pogue, M.G.; Thorington, R.W.; Vari, R.P.; Weitzman, M.J.; Weitzman, S.H. 1998. Amazon biodiversity: Assessing conservation priorities with taxonomic data. Biodiversity and Conservation 7: 1577-1587.

Magalhães, L.E.; Bjornberg, A.J.S. 1957. Estudo da genitalia masculina de Drosophila do grupo saltans (Diptera). Rev. Brasil. Biol. 17: 435-450.

Magalhães, L.E.1962. Notes on the taxonomy, morphology, \& distribution of the saltans group of Drosophila, with descriptions of four new species.Univ. Texas Publs. 6205: 135-154.

Malogolowkin, C. 1952.Sobre a genitalia dos "Drosophilidae" (Diptera). III. Grupo willistoni do genero Drosophila. Rev. Brasil. Biol. 12: 79-96.

Martins, M.B. 1989. Invasao de fragmentos florestais por especies oportunistas de Drosophila (Diptera, Drosophilidae). Acta Amazonica. 19: 265-271.

Martins, M.B. 2001. Drosophilid Fruit-Fly Guilds en Forest Fragments. In: Bierregaard, R.O. et al. (eds), Lessons from Amazonia. The ecology \& conservation of a fragmented forest. Yale University Press, New Haven \& London. p. 175-186.

McGeoch, M.A. 1998. The selection, testing and application of terrestrial insects as bioindicators. Biological Review. 73: 181201.

Medeiros, H. F.; Klaczko, L. B. 1999, A weakly biased Drosophila trap. Drosophila Information Service 82: 100-102.

Pitman, N. C. A.; Terborgh, J. W.; Silman, M. R.; Núñez, V. P.; Neill, D. A.; Cerón, C. E.; Palacios, W. A.; Aulestia, M. 2001. Dominance and distribution of tree species in upper Amazonian terra firme forests. Ecology, 82: 2101-2117.
Rafael V.; Arcos G. \& Arcos L. 2000. Ecología y Distribución del género Drosophila en Guayllabamba y el Quinche, provincia de Pichincha-Ecuador. Revista de la Pontificia Universidad Católica del Ecuador 65: 130-155.

Rafael, V.; Vela, D. 2000. Drosophila distribution in Ecuador. Drosophila Information Service, 83: 85-88.

Shorrocks, B. 1972. Invertebrate Types. Drosophila. Ginn \& Company, London, England. 144 pp.

Steege, H. ter; Pitman, N.C.A.; Sabatier, D.; Castellanos, H.; van der Hout, P.; Daly, D.C.; Silveira, M.; Phillips,O.; Vasquez, R.; van Andel, T.; van Duivenvoorden, J.; . de Oliveira, A.A; Ek, R.C.; Lilwah, R.; Thomas, R.A.; van essen, J.; Baider, C.; Maas, P.J.M.; Mori, S.A.; Terborgh, J.; Nunez-Vargas, P.; Mogollon H.; Morawetz, W. 2003. A spatial model of tree $\alpha$-diversity and $\beta$-density for the Amazon Region. Biodiversity and Conservation 12: 2255-2276.

Stotz, D.F.; Fitzpatrick, J.W.; Parker T.E. III; Moskowitz, D.K. 1996. Neotropical Birds, Ecology and Conservation. Chicago: University of Chicago Press, Chicago USA. 502pp.

Tidon, R. 2006. Relationships between drosophilids (Diptera, Drosophilidae) and the environment in two contrasting tropical vegetations. Biol. J. Linnean Soc. 87: 233-247.

UICN.2006. UICN Red list of untreatened species (http://www.iucn. org). Acceso 28/03/2006.

Val, F.C. 1982 The male genitalia of some Neotropical Drosophila: Notes \& illustrations. Pap. Avul. Sao Paulo.34: 309-347.

Valencia, R.; Foster, R.B.; Villa, G.; Condit, R.; Svenning, J.C.; Hernández, C.; Romoleroux, K.; Losos, E.; Magård, E.; Balslev, H. 2004. Tree species distributions and local habitat variation in the Amazon: large forest plot in eastern Ecuador. Journal of Ecology, 92 (2): 214-229.

van der Linde, K. 1997. Exploring the use of the Drosophila genus as biodiversity indicator. In: Guzman RS, de Groot WT (eds) Research for the Sierra Madre Forest. CVPED, Garita Heights Cabagan. Philippines.

van der Linde, K.; Sevenster, J.G. 2002. Drosophila diversity over a disturbance gradient. Proc. Sect. Exp. Appl. Ent. Netherlands Ent. Soc. 13: 51-56.

Vilela, C.R. 1983. A revision of the Drosophila repleta species group (Diptera, Drosophilidae). Rev. Bras. Ent. 27:1-114.

Young, B.E.; Stuart S.N.; Chanson J.S.; Cox N.A.; Boucher T.M. 2004. Disappearing Jewels: The Status of New World Amphibians. Arlington. Nature Serve Texas, USA. 54pp.

Recebido em 04/02/2009

Aceito em 11/05/2009 